\title{
Sistema de Información para Proyectos de Telemedicina
}

Information System for Telemedicine Projects

Fecha de recepción: $\quad 30$ de marzo de 2007

Fecha de aceptación: 13 de julio de 2007

Lilia E. Aparicio P.* Paúlo C. Coronado S.**

\section{RESUMEN"}

Este artículo muestra una visión general de los resultados obtenidos en la investigación proyectiva para el desarrollo de un sistema de información que apoya la planeación de proyectos de Telemedicina en el Sistema de Gestión de Proyectos de Telemedicina y Telesalud de Colombia: En primera instancia, la investigación involucra hospitales públicos de Bogotá para caractérizar sus redes de comunicaciones e identificar los problemas principales que hay alrededor de los sistemas de información y servicios de intercambio de datos, con el objetivo de ofrecer soporte al desarrollo de proyectos de Telemedicina. La plataforma de comunicaciones prevista para el despliegue del sistema se ofrece por la Compañía de Telecomunicaciones de Bogotá, ETB, y su modelo está fundamentado en la filosofía del software libre. La primera versión se convierte en un recurso público de información que ofrece seis subsistemas básicos: entidades de salud, operadores de telecomunicaciones, tecnologías de interconexión, servicios médicos, equipos de medicinà y proyectos en telemedicina. El sistema se apoyá en el concepto dé convergencia de redes como herramienta de desarrollo pará la solución de problemas de impacto social.

* Universidad Distrital Francisco José de Caldás medicina@udistrital.edu.co

** Universidad Distrital Francisco José de Caldas pcoronado@udistrital.edu.co 


\section{Palabras clave}

Telemedicina, sistema de información, proyectos de Telemedicina, Telesalud.

\section{ABSTRACT}

This article shows a general vision of the research and development of an Information System for Telemedicine and E-health Management Projects in Colombia. In the first place the research has been made in all public hospitals in Bogotá. The work was developed with the aim of identify the main problems that there are around the information systems and interchange services between hospitals and Communications Companies in order to give an effective support to Telemedine Projects. The platform of communications foreseen for the development is offered by the Company of Telecommunications of Bogotá, ETB; the application was released as free software. The first solution includes six subsystems: Health entities, telecommunications operators, interaction between technologies, medical services, equipment and technology, projects and organizations, where is very important the electronic data interchange to give integration of services and the convergence of networks like development tools for the solution of problems of social impact.

\section{Key words}

Telemedicine, Information System, Telemedicine Projects, Telehealth.

\section{INTRODUCCIÓN}

Con el desarrollo del proyecto SITEM (Sistema de Información para proyectos de Telemedicina), el grupo de investigación en Telemedicina de la Universidad Distrital Francisco José de Caldas (GITEM), se pone a la vanguardia en el desarrollo de proyectos basados en tecnologías de la información que integren en un único marco de trabajo la información de diferentes elementos constituyentes de las redes de telemedicina. Los resultados de la investigación preliminar [1][2] ofrecen un modelo para apoyar la solución al problema integral de salud con el objeto de migrar hacia soluciones que mejoren la cobertura y atención en servicios primarios y especializados de salud. 
Con la puesta en marcha del proyecto se pretende crear un puente entre la información comercial, la técnica y los datos fuente de investigación, basándose en los amplios y exhaustivos estudios realizados en lá ciudad de Bogotá [2]. Una vez alimentados los datos, se contará con un sistèma que permite deducir el estado actual de las redes hospitalarias cuyo fundamento teórico de estudio puede replicarse a otras ciudades.

\section{PROYECTO SITEM. SISTEMA DE INFORMACIÓN PARA PROYECTOS DE TELEMEDICINA}

SITEM es un Portal Web especializado en la gestión de datos e información de diferentes componentes estructurales de los sistemas de telemedicina [9]. Provee un ambiente de apoyo a las tareas de las comunidades de práctica involucradas en la investigación, el diseño, el mantenimiento, el desarrollo y la implementación de redes de telemedicina. Tuvo su génesis conceptual en 2000 durante el marco de la primera fase del Proyecto Telemedicina Bogotá como solución a la necesidad de administrar los resultados del estudio de campo realizado en las entidades e instituciones de salud y los operadores de telecomunicaciones de la ciudad de Bogotá [2].

Su principal objetivo es apoyar las actividades básicas de los denominados trabajadores del conocimiento [8] en el área de la telemedicina, Ofreciéndoles un repositorio de datos y herramientas que facilitan las tareas de capturar, extraer, organizar, analizar, encontrar, sintetizar, distribuir y compartir información y conocimiento. El ideal es actualizar el estado de ciertos nodos interesantes del Sistema de Salud de Bogotá, Distrito Capital, haciendo énfasis en la posibilidad de interacción nacional e internacional y en los requerimientos de las diferentes entidades que participan en el proyecto de Telemedicina auspiciado por el grupo GITEM. La figura 1 muestra la imagen que a partir del 2007. identifica al sistema en Internet ${ }^{1}$.

Con el SITEM se disminuye el tiempo de consecución, análisis y despliegue de la información. Su construcción ha sido guiada por adaptaciones de procesos

\footnotetext{
1 http://gitem.udistrital.edu.co/sitem/ Dirección de acceso en Internet al portal del Sistema de Información para proyectos de Telemedicina.
} 
de desarrollo ampliamente conocidos [5] [6] [7], siguiendo el paradigma de la orientación a objetos, lo que garantiza su facilidad de mantenimiento, escalabilidad e, indirectamente, su permanencia en el medio. En su más reciente versión explora la integración de Agentes notificadores y de Recomendación que analizan constantemente los repositorios de información para, a partir de un proceso de minería de datos, desplegar información referenciada y adaptada a las necesidades y perfiles de cada uno de los usuarios registrados dentro del sistema.

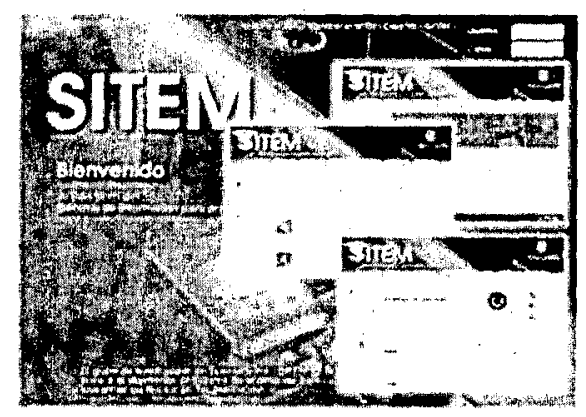

Figura 1 Interfaz Gráfica del Portal SITEM.

Propone un mecanismo para la integración de actores en el área del despliegue de soluciones de Telemedicina proveyendo un escenario ubicuo basado en tecnologías de la información y un modelo de trabajo colaborativo en red que propende a la construcción evolutiva de una base de información y conocimiento que, asociados a los conceptos de libertad en el uso de la información y el conocimiento, busca elaborar un bien público esencial para el desarrollo de la Telemedicina y la Telesalud en la sociedad.

Contiene módulos para la generación de estadísticas e informes pormenorizados de cada uno de los componentes y obteniene en pocos segundos los datos necesarios para apoyar la labor de análisis, diseño e implementación de proyectos telemédicos o de telesalud. Usa un esquema modular de crecimiento a la medida donde el esfuerzo para la creación de instrumentos nuevos de consulta se minimiza por el uso de plantillas pre - diseñadas. En lugar de ser un sistema estático, el SITEM contiene características de adaptación dinámica para cubrir las necesidades que tengan los próximos proyectos emanados del GITEM y otras entidades que hagan uso del sistema. 
El sistema gestiona la estructuración de encuestas, formularios y talleres para crear un mecanismo ágil y costo-efectivo de recolección de información para las etapas de descripción y explicación [4] en proyectos de Telemedicina. Debido a que los resultados de los análisis pueden ser compartidos, estos sirven de a las instituciones o personas que deseen realizar estudios similares acerca del tema facilitando el estudio de casos.

\section{CARACTERÍSTICAS INNOVADORAS DEL SITEM}

No son los aspectos técnicos para la creación de sistemas de información los que distinguen al SITEM sino las nuevas estructuras de información - $y$ 'su correlación, las que brindan una novedad en el contexto de la proyección de soluciones en Telemedicina. El SITEM es el primer sistema de información de acceso público en el entorno colombiano que gestiona información 'de componentes fundamentales de las redes de telemedicina. Dota a la comunidad de una herramienta única en su género, la cual puede ser utilizada como apoyo en todas las etapas de desarrollo de proyectos en telemedicina. Entre las múltiples ventajas que ofrece el sistema, facilita la obtención de información ubicua y en tiempo real, lo cuales indispensable para el soporte a la toma de decisiones.

El desarrollo mismo del SITEM se convierte en un modelo a seguir para proyectos básicos de telemedicina o telesalud orientados a la Web ya que propone una arquitectura de desarrollo interoperable, escalable y fundamentada en software libre. La integración de tecnologías Web en el SITEM brinda una plataforma robusta para la implementación de servicios en entidades de salud que carezcan de altos recursos financieros. A mediano plazo, esto podrá generar un crecimiento de la oferta de servicios de medicina a distancia[3][10], ya que ayuda a mejorar la relación costo/beneficio en proyectos que, por estar fundamentados en software comercial, han sido inviables hasta el momento.

Con la puesta en marcha del proyecto SITEM se capta la atención del usuario en salud para que conozca y entienda las posibilidades de la telemedicina y empiece a tomar un papel activo en el desarrollo y despliegue de esta opción de servicio. 


\section{ARQUITECTURA DEL SISTEMA}

SITEM es implementado sobre una arquitectura multicapa que distribuye los diferentes componentes en tres capas principales: Presentación, aplicación y datos, estando presente una capa transversal tácita de seguridad. A nivel de usuario el SITEM está compuesto por siete subsistemas autónomos, figura 2, que prestan servicios a sus pares. Estos agrupan seis componentes claves en todo proyecto de telemedicina: entidades de salud, operadores de telecomunicaciones, tecnologías de interconexión, equipos y tecnologías de captura de datos, proyectos e instituciones relacionadas con la telemedicina y servicios médicos - incluyendo módulos de vademécum, consulta de procedimientos, enfermedades y especialidades médicas.

\section{Subsistema Entidades de salud}

Este subsistema (figura 3) gestiona los datos recopilados en el estudio de campo realizado por el grupo GITEM en el marco del proyecto del Sistema de Gestión de Salud para el Distrito Capital, fases I y II. Por tanto, es el subsistema base del SITEM, y su objetivo principal es la gestión de información referente a las entidades de salud en el entorno colombiano enfocándose en tres redes principales: especialidades médicas, comunicaciones y de atención.

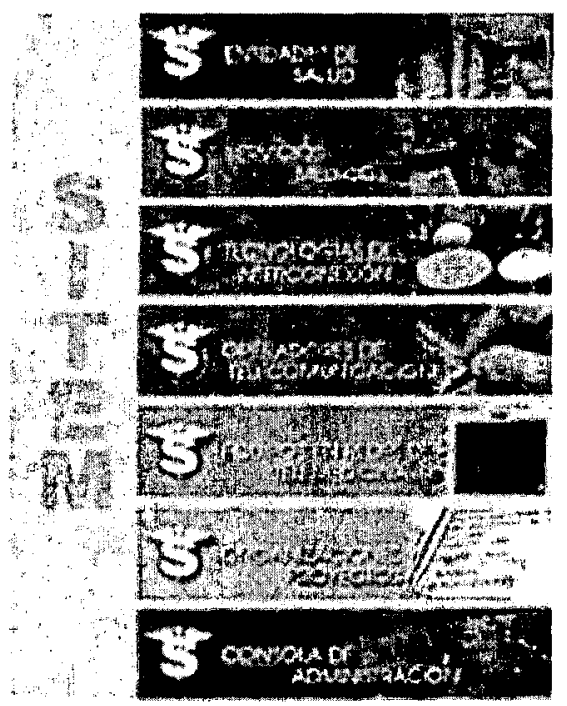

Figura 2 Componentes principales del SITEM. 
La.información disponible en el subsistema puede ser administrada por cada una de las entidades prestadoras de servicios de salud, de tal forma que se cree gradualmente un catálogo flexible para conocer el estado actual de las entidades y su potencialidad para formar parte de redes que presten servicios de salud a distancia usando TIC. Por defecto, las entidades se asocian a la arquitectura de red de la secretaría de Salud de Bogotá, pero por medio del módulo denominado redes de atención se puede crear fácilmente cualquier prototipo de red jerárquica de atención[11].

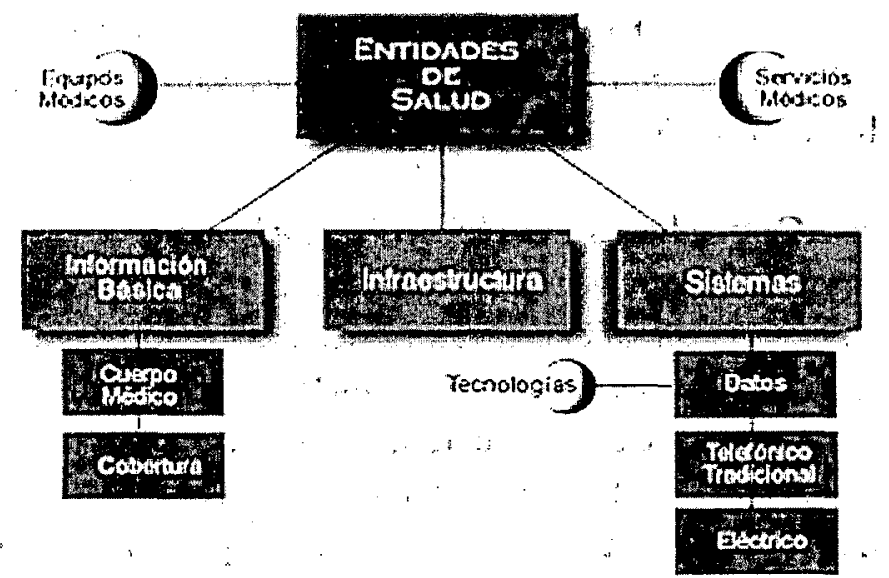

Figưra 3 Arquitectưra Básica del Subsistema Ėntidades de Salüd.

\section{Subsistema Tecnologías de interconexión}

Administra información relacionada con las tecnologías y protócolós de.interconexión disponibles en las redes de acceso y transporte. Estas tecnologías se ordenan principalmente sobre el modelo de referencia OSI, pero se puede crear desde el módulo de arquitecturas, cualquier otro tipo de modelo. En la actualidad se tiene como alternativa de clasificación el modelo de TCP/IP. Es una guía técnica que muestra la información de las capas físicas, de enlace y de red en formatos básicos - o de características generales, e intermedios - o de característiças técnicas.

El objetivo principal que se persigue con la implementación de este subsistema es proveer a los analistas información para la revisión sistemática de las diferentes opciones que brindan los fabricantes de dispositivos y así proyectar redes que sean técnicamente viables, La información se estructura de acuerdo 
con indicadores cualitativos y cuantitativos que permiten evidenciar el carácter de interoperabilidad, impacto y permanencia de la tecnología en el mercado.

\section{Subsistema Equipos y tecnologías}

Información técnica sobre los diversos equipos y tecnologías usadas en telemedicina. Posee secciones para la gestión de información especializada de proveedores y fabricantes, así como la gestión de especificaciones técnicas, funcionales y físicas de los equipos.

Combinado con los demás subsistemas, provee un mecanismo eficiente para realizar auditorías ex-ante en redes tecnológicas, valorando alternativas de intercambio y reposición en los nodos.

\section{Subsistema Operadores de telecomunicaciones}

Contiene información relacionada con operadores de telecomunicaciones, figura 4, con énfasis en las características técnicas de los servicios que ofrecen, su cobertura y tarifas. Brinda a los analistas información comparativa entre operadores, lo que permite determinar las ventajas y desventajas entre diferentes opciones de interconexión, de acuerdo con los servicios médicos que se quieren implementar. Este módulo se complementa con la información de los subsistemas de tecnologías de interconexión y servicios médicos, así como de la información de dominio público que muestra el Sistema de Información Unificado para el sector de las Telecomunicaciones mantenido por la Comisión Reguladora de Telecomunicaciones.

\section{Subsistema Organizaciones y proyectos}

Posee herramientas informáticas para la gestión de información de proyectos nacionales y de la región en el ámbito de la telemedicina, la telesalud y la tele educación en medicina.

Incluye secciones para la gestión de los datos correspondientes a organizaciones y grupos de investigación que trabajen en el área de la telemedicina. Este subsistema es uno de los pilares del SITEM, pues despliega aplicaciones que fomentan el trabajo en grupo y se realiza la captura de las experiencias adquiridas en los diferentes proyectos desarrollados en el área. El módulo posee herramientas de edición que permiten la sincronización del trabajo en la realización de estudios técnicos y de factibilidad. 


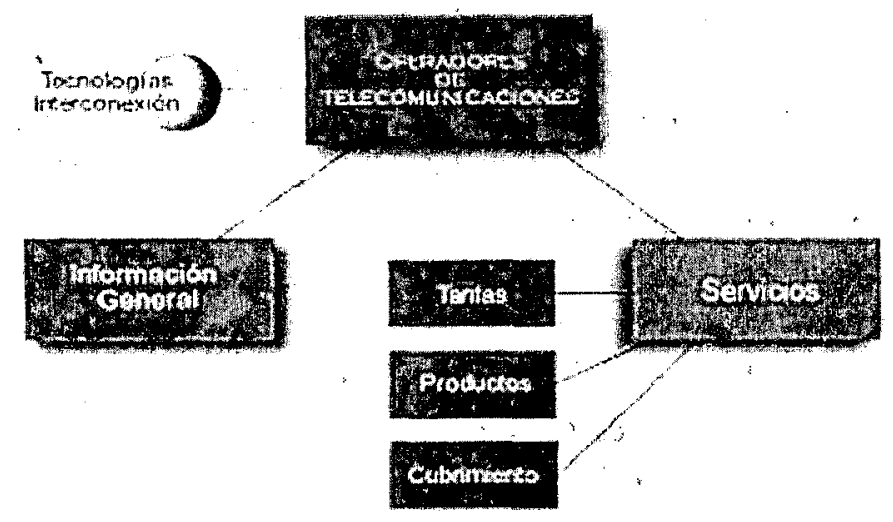

Figura 4 Componentes principales del Subsistema Operadores de Telecomunicaciones.

\section{Subsistema Servicios médicos}

Este subsistema, cuya arquitectura se muestra en la figura 5," contiene una guía catalogada de las diferentes especialidades médicas. Se pone especial atención en la descripción de los requerimientos técnicos y tecnológicos que exige cada una de ellas, así como el perfil de los profesionales y las entidades educativas de formación de especialistas. Algunos componentes de este subsistema permiten la gestión de información, con base en la normatividad colombiana e internacional, relativa a procedimientos, medicamentos, enfermedades y laboratorios. Esto los convierte en un elemento de apoyo de una plataforma de servicios, como la consulta remota, el telediagnóstico o la teleeducación médica.

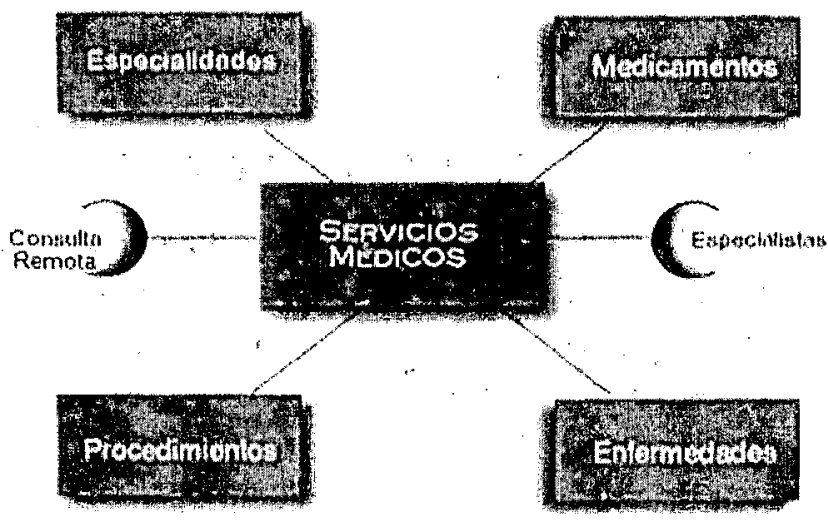

Figura 5 Subsistema Servicios médicos. 


\section{Subsistemas Entornos de aprendizaje}

Siguiendo la filosofía de integración del SITEM a proyectos de software libre, el subsistema Entornos de aprendizaje incorpora y adapta los elementos de la plataforma Moodle y provee un ambiente en línea para la estructuración, el mantenimiento y la distribución de conocimiento a través de cursos y seminarios. El subsistema permite libre acceso de usuarios a un conjunto de cursos y seminarios que apoyan el desarrollo de competencias en el área de la teleinformática, la telesalud, la telemedicina, las tecnologías de la información y las comunicaciones. Con un enfoque constructivista se procura la retroalimentación de los contenidos a la comunidad.

Como patrón de desarrollo, el SITEM integra a su arquitectura (figura 6) soluciones exitosas y robustas en el mundo del software libre. De esta forma, reutiliza gran cantidad de aplicaciones, las adapta para proveer un ambiente integrado y aumenta sus prestaciones para implementar nuevos casos de uso.

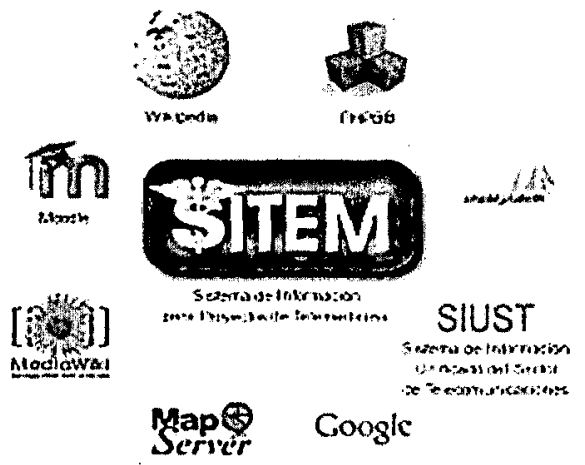

Figura 6 Herramientas públicas y de software libre que complementan al SITEM.

\section{RESULTADOS EN EL DESARROLLO DEL PROYECTO}

Cuando se propuso el proyecto de Telemedicina Bogotá, se tuvo en cuenta problemas puntuales para la implementación de servicios médicos en el Distrito Capital. Por ejemplo a) En el momento no existe un diagnóstico real sobre los servicios requeridos en el área dé telemedicina, razón suficiente para iniciar un trabajo de campo que establezca la situación actual de servicios médicos y la demanda real, así como la posibilidad de conocer a corto, mediano y largo plazos cuáles serían los costos de inversión que permitirían dar 
soluciones al problema de cobertura se servicios de salud.b) La socialización del conocimiento alrededor de las tecnologías aplicadas al desarrollo de la medicina es uno de los valores que lleva al éxito de soluciones efectivas en el sector salud; por tal motivo es necesario desarròllär un plan de alfabetización en el sector salud y en el sector gubernamental y académico. c) En el país no existen estrategias de investigación en esta área del conocimiento para llevar a cabo un estudio real que permita dar el paso a soluciones verdaderas sobre desarrollo tecnológico o experimental para implementar centros de investigación en telemedicina.

Buscando suplir estas debilidades, se emprendió la tarea de recopilar la información de los diferentes entes que conformarían una red de Telemedicina. Se creó el proyecto SITEM y, en las fases I y II se diseña y se hace una prueba de funcionalidad. El beneficio de oportunidad como proyecto para una investigación valiosa en el tema del desarrollo de software distribuido, interoperable, robusto y basado en software libre, en la que además se entra en fase piloto para depuración de la arquitectura, la depuración de los subsistemas estructurales y la inclusión de la base de información. La fase IVse relaciona específicamente con la migración de la solución a plataformas diferentes a la usada en la etapa de prueba piloto con él ánimo de capturar un mayor ámbito del mercado en servicios de telemedicina y telesalud.

\section{BIBLIOGRAFÍA}

[1] Aparicio, L. y Ramírez, J., Arquitectura de red de telemedicina, Bogotá, Centro de Investigaciones y Desarrollo Científico, Universidad Distrital FJC, 2003.

[2] Aparicio, L. Propuesta de estudio red de telemedicina Bogotá, BogotáGrupo GITEM, Universidad Distrital FJC, 2000.

[3] Craig. J., Patterson, V., "Introduction to the practice of telemedicine". Journal of Telemedicine and Telecare. 2005. Pp. 3-9.

[4] Hurtado, J., Metodología de la investigación Holistia., Caracas, Venezuela, Sypal 2000. 
[5] Jacobson, I., Booch, G. y Rumbaugh, J., El proceso unificado de desarrollo de software, Madrid, Addison-Wesley 2000.

[6] Koch, S., FreeOpen Source Software Development, Idea Group, 2005.

[7] Larman, C. Agile and iterative development: a manager 's guide, Addison Wesley, 2004.

[8] Nonaka, I. A., Dynamic theory of organizational knowledge creation, Organization Science, pp. 14-37.

[9] Salazar, J. y Kopec, A. Aplicaciones de telecomunicaciones en salud en la subregión Andina - Telemedicina, Organismo Andino de Salud, OPS, 2002.

[10] ITU. Telemedicine and Developing Countries-Lessons Learned, Document 2/116-E. ITU-D STUDY GROUPS. Question 14/2: Fostering the application of telecommunication in health care. Identifying and documenting success factors for implementing telemedicine.

[11] Yellowlees, P.M., "Successfully developing a telemedicine system". Journal of Telemedicine and Telecare, 2005, pp 331-335. 